\title{
An optical flatness measurement system for medium-sized surface plates
}

\author{
Kuang-Chao Fan and Fang-Jung Shiou \\ Department of Mechanical Engineering, National Taiwan University, Taipei \\ Taiwan, ROC
}

This paper describes the work to develop a parallel beam scanning system (PBSS) for the noncontact measurement of surface flatness of such medium-sized surface plates, as sheet metals, sheet-moulding compound (SMC) plates, and glass plates, which are difficult to measure by traditional methods. The PBSS consists of a He-Ne laser source with good pointing stability, a scanner to create divergent scanning beams, a large aplanatic meniscus lens to convert the divergent beams to parallel beams, a linear stage to drive the testpiece to each sampling position, a screen for the projection of reflected beams from the tested surface, and an image-processing unit to analyze the projected image. Because of the out-of-flatness of the surface, the straight line formed by the incident parallel beams will be distorted and magnified on the screen as it is reflected from the surface. A chargecoupled-device (CCD) camera is employed to capture the image of the distorted line each time, and proceeds in line-by-line sequence. With the proposed mathematical model, the three dimensional (3-D) flatness data of the testpiece can be converted from the input image data and analyzed by the least-squares method. Experimental results by the use of this system have shown good agreement with the results obtained from a coordinate measuring machine (CMM). Because of the limit of the meniscus lens, the workpiece size is within $200 \mathrm{~mm}$ in width. (C) 1997 Elsevier Science Inc.

Keywords: surface plate; flatness measurement; scanning system

\section{Introduction}

The definition of flatness error of any surface plate has been specified in many different standards on the geometric tolerance, ${ }^{1-3}$ which relates to the geometric quality of products. Many technical methods have been proposed for measurement of surface flatness according to the size and the quality of the surface to be inspected. For big surface plates, such as granite tables, the flatness measurement can be carried out using an electronic level, ${ }^{4,5}$ autocollimator, ${ }^{6}$ or laser interferometer. ${ }^{7}$ For small surfaces, such as gauge

Address reprint requests to Professor Kuang-Chao Fan, Department of Mechanical Engineering, National Taiwan University, Taipei, Taiwan, ROC. E-mail: fan@ccms.ntu.edu.tw blocks and mirrors, the flatness error can be measured by interferometry. ${ }^{8}$ However, for mediumsized plates all the above methods are not applicable, because the plate is distorted by the weight of the instrument placed on it, or its size is too big for the interferometer field of view. Some traditional methods that might be used for the flatness measurement of surface plates include the projection of grid patterns ${ }^{9}$ and the digitizing with lowforce linear transducers. ${ }^{10,11}$ The former cannot provide sufficient resolution and sensitivity, while the latter is time consuming and requires an expensive positioner, such as precision $X-Y$ table, or coordinate measuring machine (CMM).

Other researchers have shown that, with the scanning light technique, the surface quality of sheet-moulding compound (SMG) plates can be 


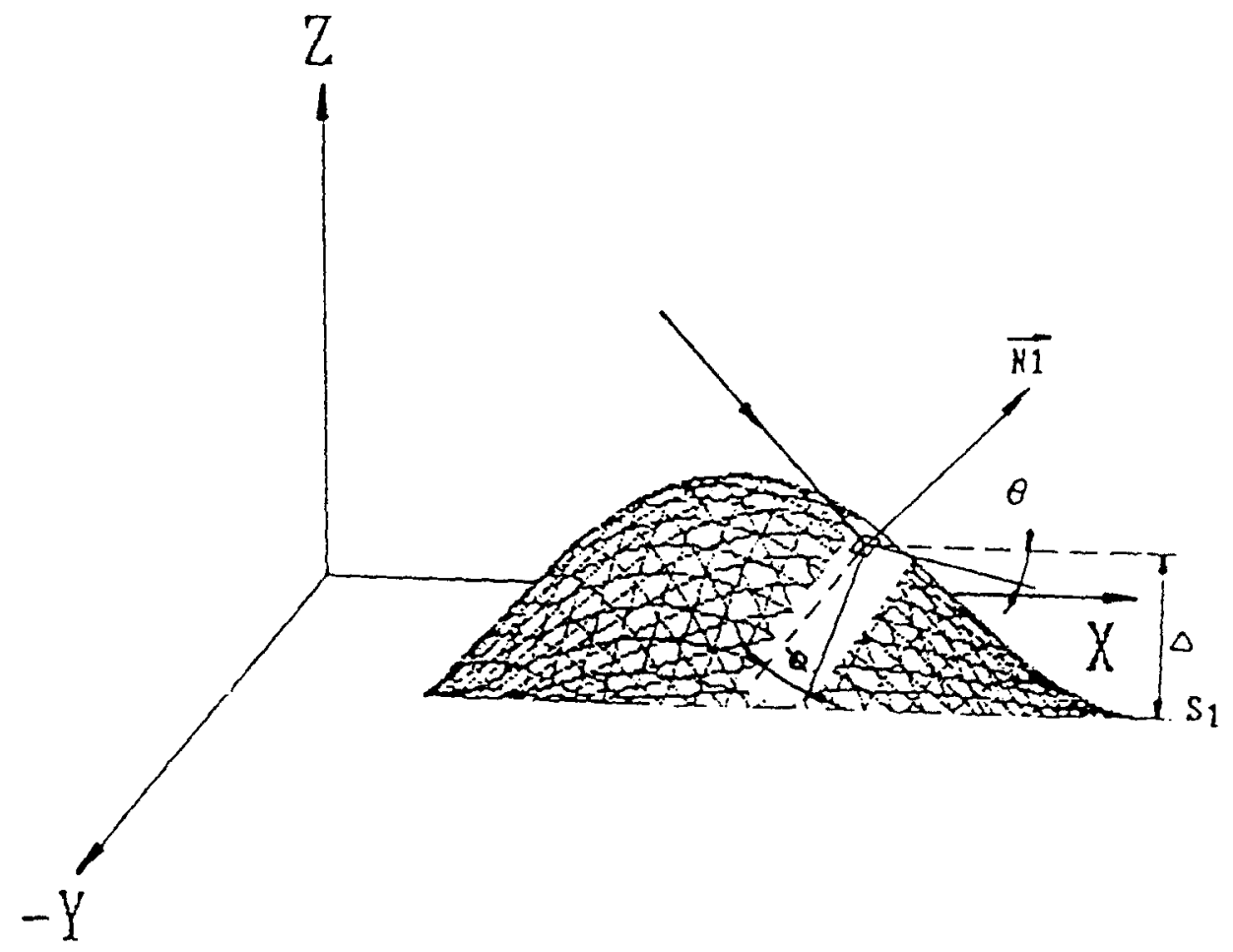

Figure 1 Three-dimensional light-scattering diagram (exaggerated in height)

evaluated quantitatively. ${ }^{12-15}$ They are, however, only able to obtain the approximate flatness by means of an index. The true surface profile still cannot be explored.

This article describes the newly developed parallel beam scanning system (PBSS) that can measure the real flatness profiles of most kinds of surface plates, where the reflectivity of the inspected surface is good. In addition, a mathematical model is proposed for the analysis of measured image data. The primary concept of this model is to obtain the slope changes of the measured surface and then convert the slopes to height differences, rather than to deliberately get the variation in the surface heights directly, as in some traditional methods. ${ }^{9-11}$ Experimental results showed that the surface profile as well as the flatness error of the inspected plates were in good agreement with the results obtained by the CMM. Because of the size restriction of the large aplanatic meniscus lens, the width of the inspected surface is limited to $200 \mathrm{~mm}$.

\section{Mathematical model of light reflection principle}

A laser beam is projected onto the work surface along a vector with constant angle $\theta$, reflected from the surface along the specular direction, and projected on a screen a certain distance away. This reflected beam will vary its position on the screen with the actual height $\Delta$ of the incident point on the surface and also with its slopes in two principal directions ( $\theta$ and $\phi)$, as shown in Figure 1. Accordingly, this projected point on the screen will be affected by these three variables; i.e., $\Delta, \theta$, and $\phi$. The position of the projected spot on the screen can be analyzed with respect to these three factors.

\section{Influence of the screen spot by surface height}

As seen in Figure 2 (which is exaggerated in its surface height), when the surface height moves from $S 1$ plane to $S 2$ plane by a distance $\Delta$, the spot

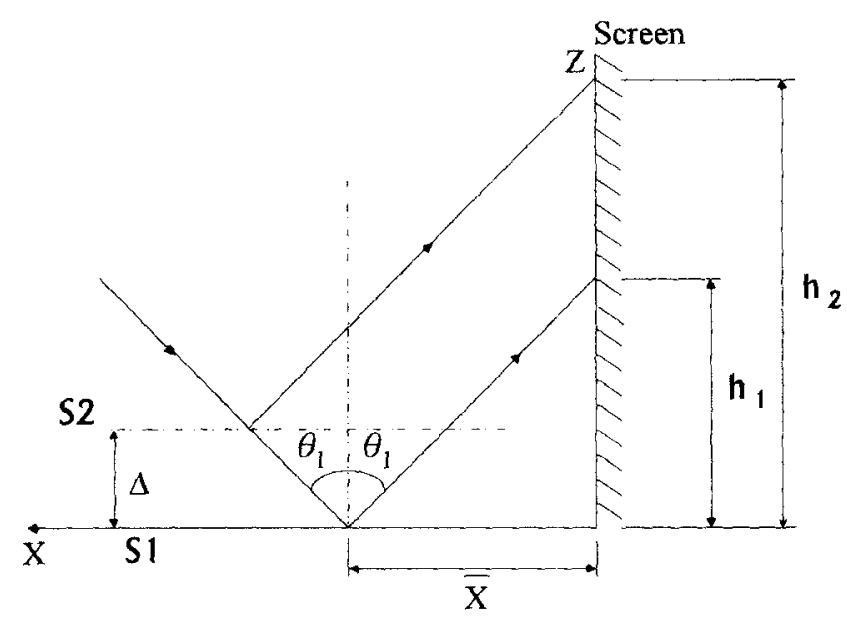

Figure 2 Relation between the surface height and the reflected beam 
Fan and Shiou: An optical flatness measurement system

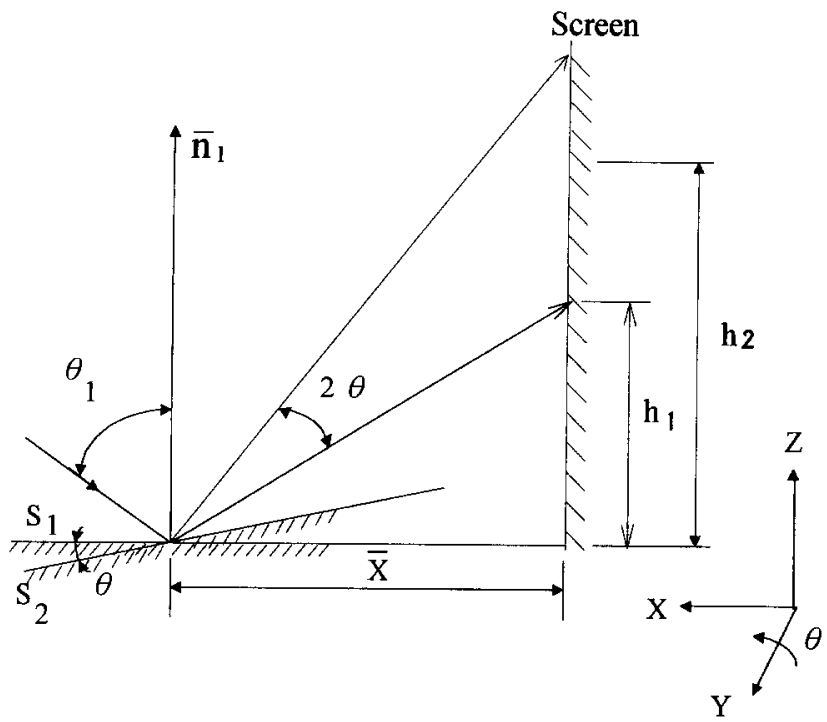

Figure 3 Relation between the surface slope in the $X$-direction and the reflected beam

of the reflected beam on the screen will shift its the vertical position by

$$
\begin{aligned}
\delta h_{1} & =h_{2}-h_{1} \\
& =(\bar{x}+\Delta \tan \theta) \cot \theta+\Delta-\bar{x} \cot \theta \\
& =2 \Delta
\end{aligned}
$$

where $\bar{x}$ indicates the distance of the screen from the incident point on the surface.

\section{Influence of the screen spot by the surface slope in $x$-direction}

As seen in Figure 3, when the surface tilts from $S 1$ plane to $S 2$ plane in the $X$-direction by an angle $\theta$, the reflected beam will tilt $2 \theta^{\circ}$. The spot will move from its original height $h_{1}$ to height $h_{2}$ on the screen. Thus, the spot elevates by

$$
\delta h_{2}=h_{2}-h_{1}=\bar{x}[\cot (\theta-2 \theta)-\cot \theta]
$$

Influence of the screen spot by the surface slope in $y$-direction

As seen in Figure 4, when the surface tilts in $Y$ direction by an angle $\phi$, the reflected beam will rotate to the $\mathbf{w}$ direction with respect to the new surface normal $\mathbf{n}_{1}$. Some notations are defined as follows.

$S 1$ : the reference $X-Y$ plane.

$S 2$ : the new plane tilted from $S 1$ by angle $\phi$ (not shown in the figure)

$\theta$ : the incident angle of laser beam

p : the unit vector of incident laser beam

$\mathbf{w}$ : the unit vector of reflected laser beam

n : the unit vector of reference plane $S 1$

$n_{1}$ : the unit vector of tilted plane $S 2$

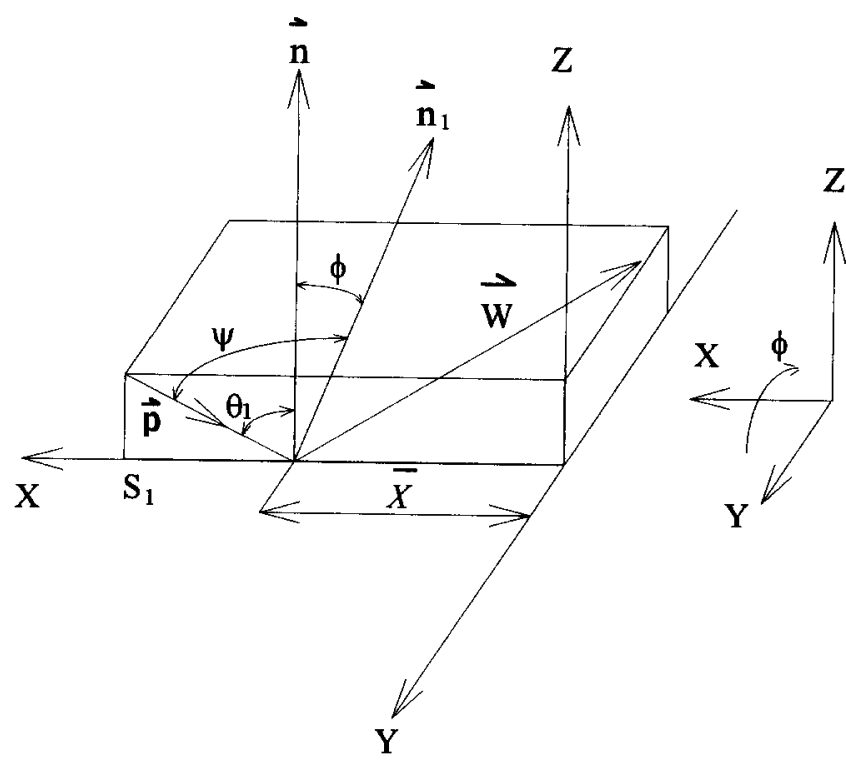

Figure 4 Relation between the surface slope in the $Y$-direction and the reflected beam

$\psi$ : the angle between $\mathbf{n}_{1}$ and $\mathbf{p}$

The unit vectors can be expressed in the following forms.

$\mathbf{p}=(-\sin \theta, 0,-\cos \theta)$

$\mathbf{n}=(0,0,1)$

$\mathbf{n}_{1}=(0, n 2, n 3)$

$\mathbf{w}=(w 1, w 2, w 3)$

where $n 2, n 3$ are the directional cosines of $\mathbf{n}_{1}$, and $w 1$, $w 2, w 3$, are directional cosines of $\mathbf{w}$. From Figure 4, the following relations exist.

$$
\begin{aligned}
-\mathbf{p}+\mathbf{w} & =2 \cos \psi \mathbf{n}_{1} \\
-\mathbf{p} \cdot \mathbf{n}_{1} & =\cos \psi=\langle\sin \theta, 0, \cos \theta\rangle \cdot\langle 0, n 2, n 3\rangle \\
& =\cos \theta n 3 \\
\mathbf{n} \cdot \mathbf{n}_{1} & =\langle 0,0,1\rangle \cdot\langle 0, n 2, n 3\rangle \\
& =n 3=\cos \phi
\end{aligned}
$$

Substituting Equation (5) into Equation (4) yields

$$
\cos \psi=\cos \phi \cos \theta
$$

Therefore, the components of $w$ can be obtained from Equation (3) as

$$
\begin{aligned}
& w 1=-\sin \theta \\
& w 2=2 \cos \phi \cos \theta n 2 \\
& w 3=-\cos \theta+2 \cos \theta \cos ^{2} \phi
\end{aligned}
$$

Let the incident point of $\mathbf{p}$ on the work surface be $P\left(x_{1}, y_{1}, z_{1}\right)$ and the projected point of $w$ on the screen be $Q\left(X_{1}, Y_{1}, Z_{1}\right)$. The line representing the reflected beam has the following relations. 

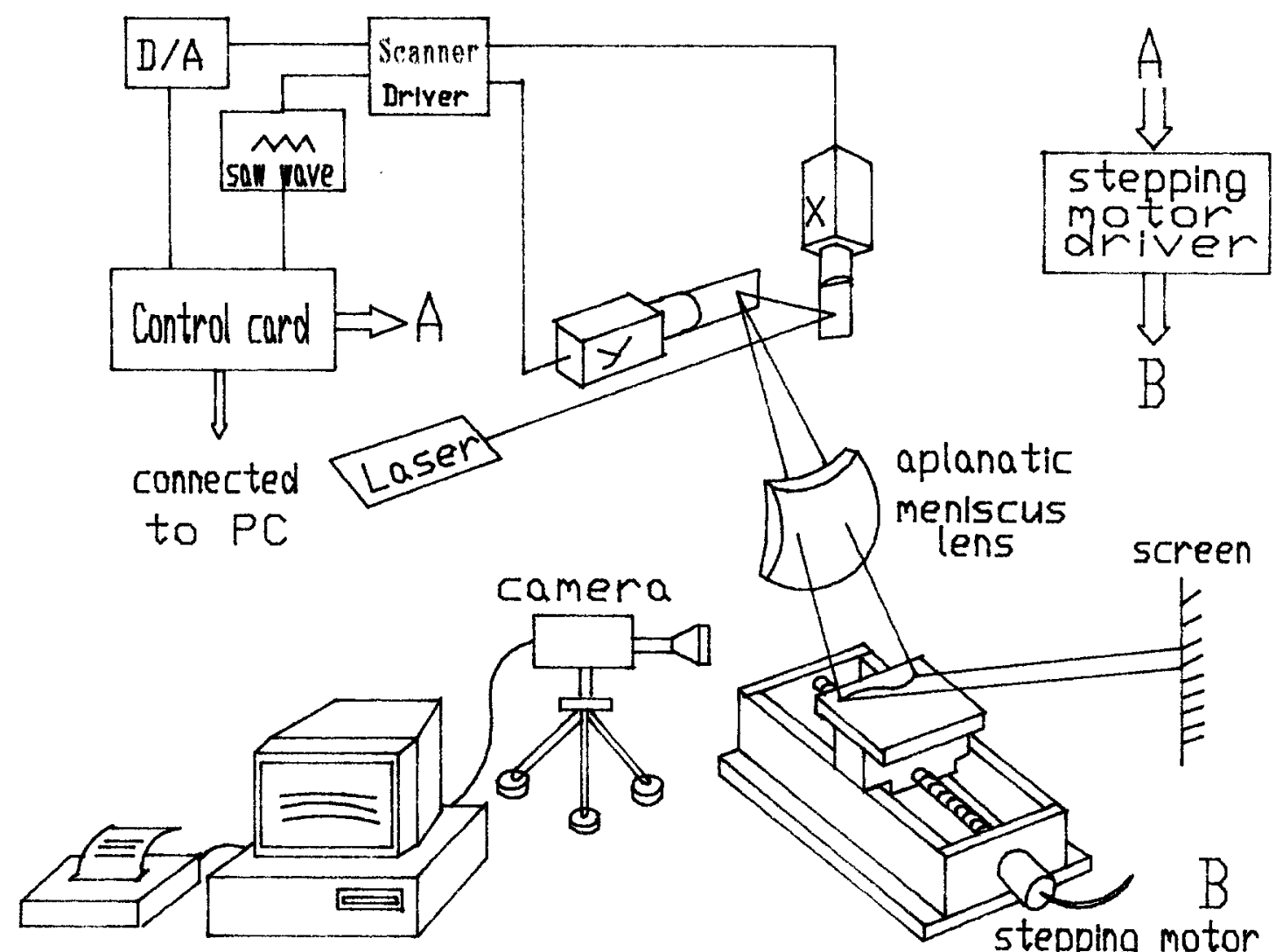

Figure 5 Schematic diagram of the PBSS setup

$$
\frac{X_{1}-x_{1}}{w_{1}}=\frac{Y_{1}-y_{1}}{w_{2}}=\frac{Z_{1}-z_{1}}{w_{3}}=T
$$

From Figure 4, it is clearly seen that $x_{1}=\bar{x}$, and $y_{1}$ $=z_{1}=X_{1}=0$. Equation (8) can, therefore, be simplified to

$$
T=\frac{\bar{x}}{\sin \theta_{1}}
$$

and

$$
\begin{aligned}
Z_{1} & =w_{3} T \\
& =\left(2 \cot \theta \cos ^{2} \phi-\cot \theta\right) \bar{x}
\end{aligned}
$$

The variation in height of the projecting spot on the screen due to the slope change $\phi$ of the work surface in the $Y$-direction can, therefore, be obtained from Equation (9) and Figure 2 as

$$
\delta h_{3}=Z_{1}-h_{1}=-2 \bar{x} \cot \theta \sin ^{2} \phi
$$

\section{Overall displacement of projecting point on the screen}

The overall vertical displacement of the projecting point on the screen caused by the height change, the slope change in $X$-direction, and the slope change in $Y$-direction of the work surface will be the sum of Equations (1), (2), and (10).

$$
\begin{aligned}
\Delta H= & \delta h_{1}+\delta h_{2}+\delta h_{3} \\
= & 2 \Delta+\bar{x}[\cot (\theta-2 \theta)-\cot \theta \\
& \left.-2 \cot \theta \sin ^{2} \phi\right]
\end{aligned}
$$

\section{Measurement System of PBSS}

The proposed mathematical model requires two constant conditions for all measured points; i.e., the constant incident angle of the laser beams $\theta_{1}$ and the constant distance between the measured point and the screen $\bar{x}$. The hardware design of the measurement system should conform to these two conditions. To ensure the constant angle $\theta_{1}$, the generation of parallel laser beams is necessary. The second condition requires that the scanner generates only one scanning line on the inspected surface, and this scanning line must be parallel to the screen with a constant distance $\bar{x}$. To keep this distance constant, the plate has to be moved step-by-step by a linear stage. Traditional methods using a $X-Y$ scanner to scan the laser beams on the whole fixed surface do not meet these conditions. This is the special feature of the PBSS design.

As seen in Figure 5, the PBSS, consists of a 5-mw He-Ne laser source with good pointing stability, a scanner to create divergent scanning 
Fan and Shiou: An optical flatness measurement system

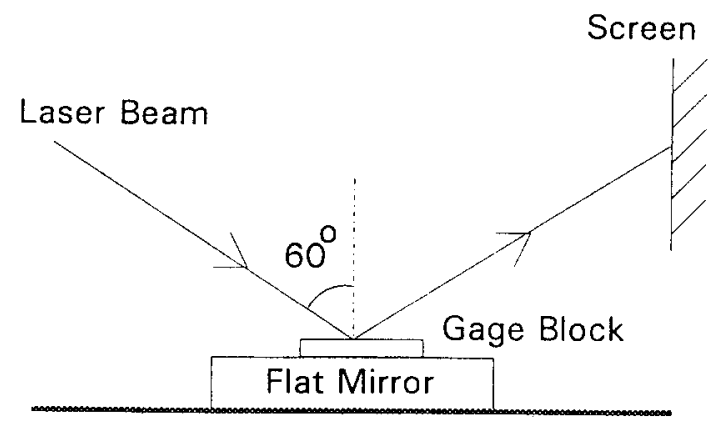

Sensitivity test of PBSS with gage blocks

Figure 6 Sensitivity test of change of height by PBSS with gauge blocks

beams, a large aplanatic meniscus lens to convert the divergent beams to parallel beams with minimum spherical aberration or coma, a linear stage to drive the specimen to each sampling position, a screen for the projection of reflected beams from the tested surface, and an image-processing unit to capture and analyze the projected image. The $Y$ scanner used in the PBSS provides the initial position adjustment of the $X$-scanning beams. Because of out-of-flatness of the inspected surface, the straight line formed by the incident parallel beams will be distorted and magnified on the screen as it is reflected from the surface. Initial adjustment of the light incident angle $\theta_{1}$ is very critical to ensure the continuity of the reflected line on the screen. A charge-coupled-device (CCD) camera is used to capture the image of this distorted line each time. The linear stage then positions the specimen step-by-step to carry out measurements line-by-line throughout the longitudinal length of the surface. Having collected all the image lines, the computer will relocate the same position of each image line on the monitor as it is originally appeared on the work surface. Because the diameter of the meniscus lens used in this PBSS is $200 \mathrm{~mm}$, it restricts the width of the parallel beams as well as the surface width.

\section{Digital image-processing technique}

The captured image of distorted lines must be processed prior to the computation of flatness errors. The frame of the graphic monitor is composed of $512 \times 512$ pixels. Digitized data are represented by the corresponding pixels in 256 gray levels. Each image line has a thickness that varies along the line, probably from scattering caused by the roughness of the inspected surface. ${ }^{16}$ This is, however, not the major interest of this research work. Only the degree of distortion of each image line is required to realize the actual surface profile. To achieve this, two processes are implemented.

\section{Binary contrast enhancement}

The digitized raw image data may have large variations in gray levels. We chose a simple intensity threshold operator to identify the line, with pixels below the threshold assigned to black $(0)$ and those above assigned to light (255). From this enhanced image, the length and the thickness of each line can be clearly quantified with corresponding pixel numbers. From experiments, the appropriate threshold of gray level found for this study was 30 .

\section{Image line thinning process}

After the binary coding process, each image line can be clearly seen with varying thickness along its

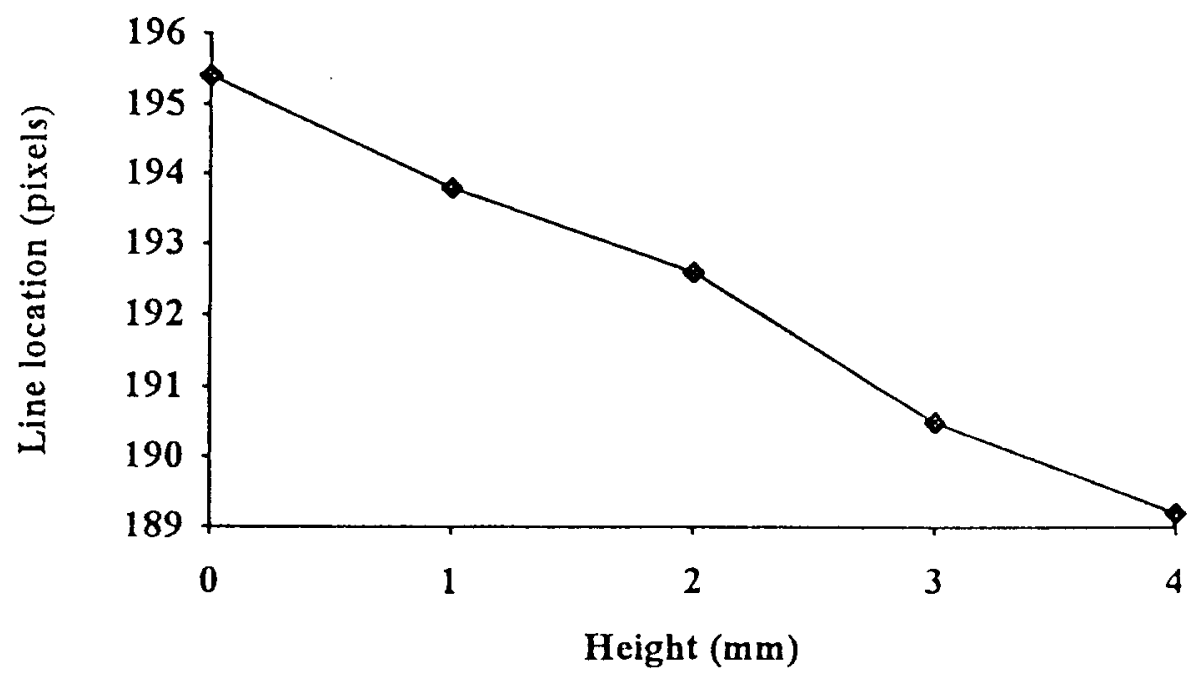

Figure 7 Results of the spot positions versus surface heights 
Fan and Shiou: An optical flatness measurement system

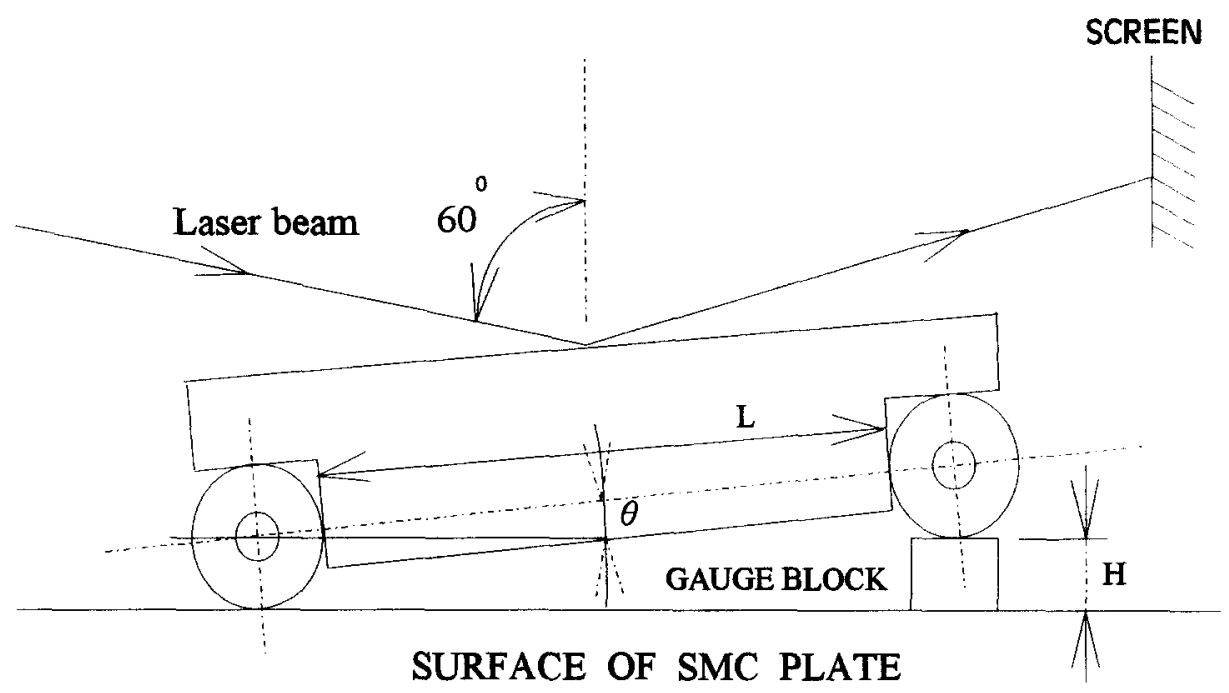

Figure 8 Sensitivity test of change of slope by PBSS with sine bar

length. At each pixel position in the horizontal direction (the $X$-direction on the monitor) of any particular line, the center of the band at that position was selected to represent the vertical position ( $Y$-position) of that point. Linking all the continuous central points a thin line can be formed. Consequently, each line can be represented by 512 points, with resolution to 1 pixel in the $X$-coordinate, and 0.5 pixel in the $Y$-coordinate if the line thickness consists of even number of pixels.

\section{Flatness analysis}

\section{Selection of a common reference line}

As described earlier in section the Measurement system..., with the light reflection model, the projected point on the screen will be affected by

\section{Slope calibration}

Sine bar center distance is $100 \mathrm{~mm}$

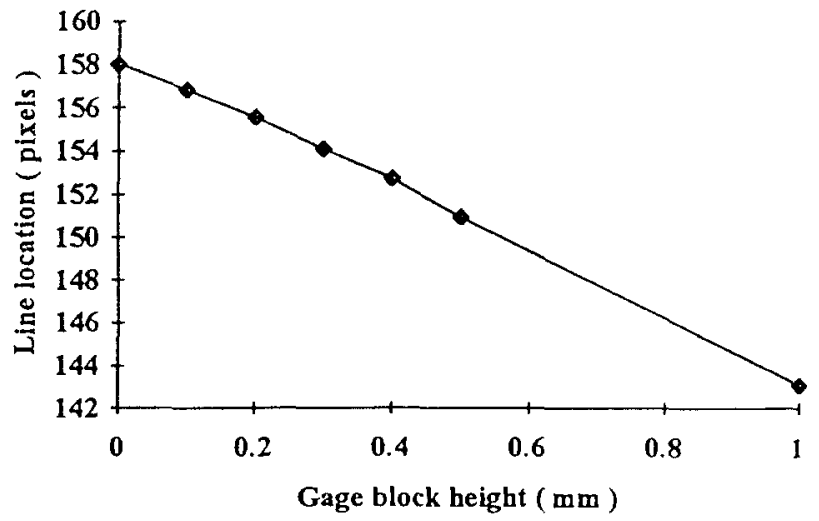

Figure 9 Results of the spot positions versus the $X$-slopes the height $\Delta$, the $X$-slope $\theta$, and the $Y$-slope $\phi$ of the corresponding measured point on the surface, as expressed by Equation (11). The total vertical displacement $\Delta H$ of that projected point can be obtained by experiment, in corresponding pixel number offset from a reference line. The vertical displacement $\Delta H$ along each individual projected line encodes the relative height changes of the sample along the illuminated line. The reference line for this analysis is arbitrary, but to refer all of the illuminated lines together and get the profile of the entire sample, the same reference line is used for image lines. In practice, this common reference line can be any line, say, the first mean line, because final leveling work (the computation of least-squares plane) still must be done at the end of flatness analysis.

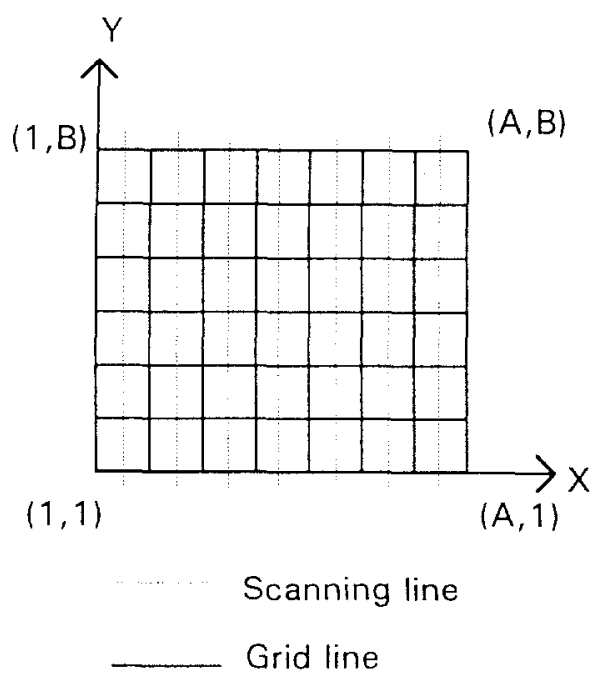

Figure 10 Rectangular grid formation of the surface 


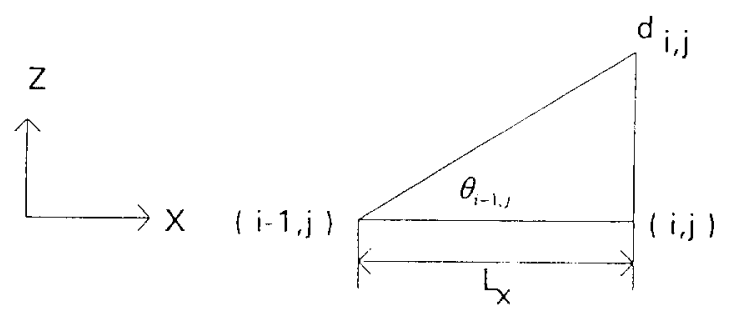

Figure 11 Relation between the surface slope and the surface height

\section{The simplified light reflection model}

In Equation (11), there are three variables to be solved; namely, $\Delta, \theta, \phi$, which cause this equation to be indeterminate. Therefore, an in-depth investigation must be carried out to simplify this model so that two less important variables can be neglected.

First, because this is the study on the flatness measurement, most of the inspected surfaces must be fairly flat and smooth. The mapping of points on the part and the screen can be assumed 1 to 1 . The second-order term $\left(\sin ^{2} \phi\right)$ in Equation (11) can be neglected, because $\phi$ must be very small. Equation (11) can thus be simplified to only two variables as

$$
\Delta H=2 \Delta+\bar{x}[\cot (\theta-2 \theta)-\cot \theta]
$$

Furthermore, two additional experiments were carried out to see the individual weights of $\Delta$ and $\theta$ in Equation (12). The first one was to change the surface height by placing different gage blocks on a flat mirror to see the variation in the projecting positions on the screen. Figure 6 shows the diagram of this experiment using PBSS setup. Figure 7, plots the tested results from which it can be seen that only 1.5 pixels movement of the screen spot was obtained per $1 \mathrm{~mm}$ change of the surface height. The second experiment was to change the $X$-slope by varying different angles of a sine bar on the PBSS setup, as shown in Figure 8. Tested results showed that 1.5 pixels movement of the screen spot could be incurred by a slope change of $0.001 \mathrm{rad}$, as seen in Figure 9. From these

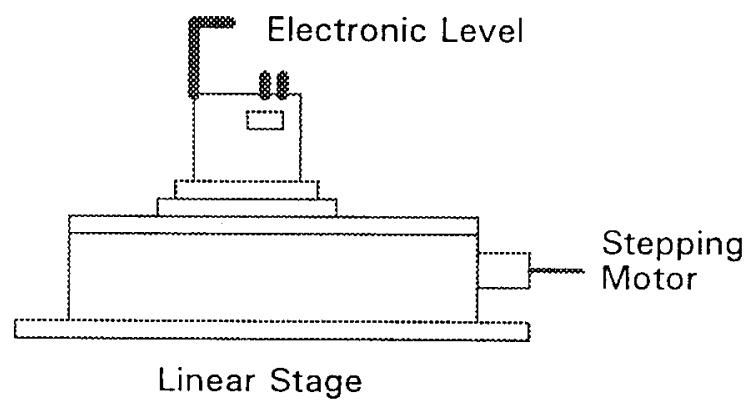

Figure 12 Pitch error calibration of the linear stage two experiments, it is apparent that the change of the $X$-slope of the inspected surface affects the pointing position on the screen more significantly than the change of the surface height. Therefore, Equation (12) can be further simplified to

$$
\Delta H=\bar{x}[\cot (\theta-2 \theta)-\cot \theta]
$$

Rewriting Equation (13) into a more general form, the surface slope in the optical incidentreflecting plane at any target position $\left(X_{i}, Y_{j}\right)$ can be obtained as

$$
\theta_{i, j}=\frac{1}{2}\left[\theta_{1}-\tan ^{-1}\left(\frac{1}{\frac{\Delta H_{i, j}}{\bar{x}}+\cot \theta_{1}}\right)\right]
$$

where $\Delta H_{i, j}$ denotes the beam spot movement on the screen with respect to the $j$ th sampling point of the $i$ th scanning line on the work surface.

\section{Measurement procedure}

From the simplified general Equation (14), it is quite clear that the principal concept of the PBSS is first to obtain the slope of the target point in the optical incident-reflecting plane, rather than the direct surface height of that point relative to a reference plane. These slope data of the entire surface can then be converted to the corresponding height data of the target points. This concept coincides with the principle of flatness measurement by angles using an electronic level, autocollimator, or laser interferometer. The total number of measured surface points obtained from the PBSS measurement process is 512 (pixel number per image line) times the number of total scanning lines. Practically, however, it does not necessarily require so many points, as long as the true surface profile can be expressed smoothly and accurately. Therefore, in this study, the inspected surface is formatted by a rectangular grid pattern of $A \times B$ nodes (a rectangular mesh) as used by other angular measurements mentioned above, as shown in Figure 10. We assume the surface heights of all points between any two consecutive nodes are linearly varied; i.e., with a constant slope. This slope can be obtained from Equation (14) by the PBSS technique. The corresponding scanning line can be arranged to project onto the center point of these two consecutive nodes. Therefore, the total scanning lines will be $A-1$ if the plate to be inspected is moved in the $X$-direction. $B$ is then the number of target points selected from each image line.

To start the measurement, the thin plate is placed on the linear stage aligned so that the scanning beams emitted from the lefthand side can fall right on the midpoint of the first two $X$ 
Fan and Shiou: An optical flatness measurement system

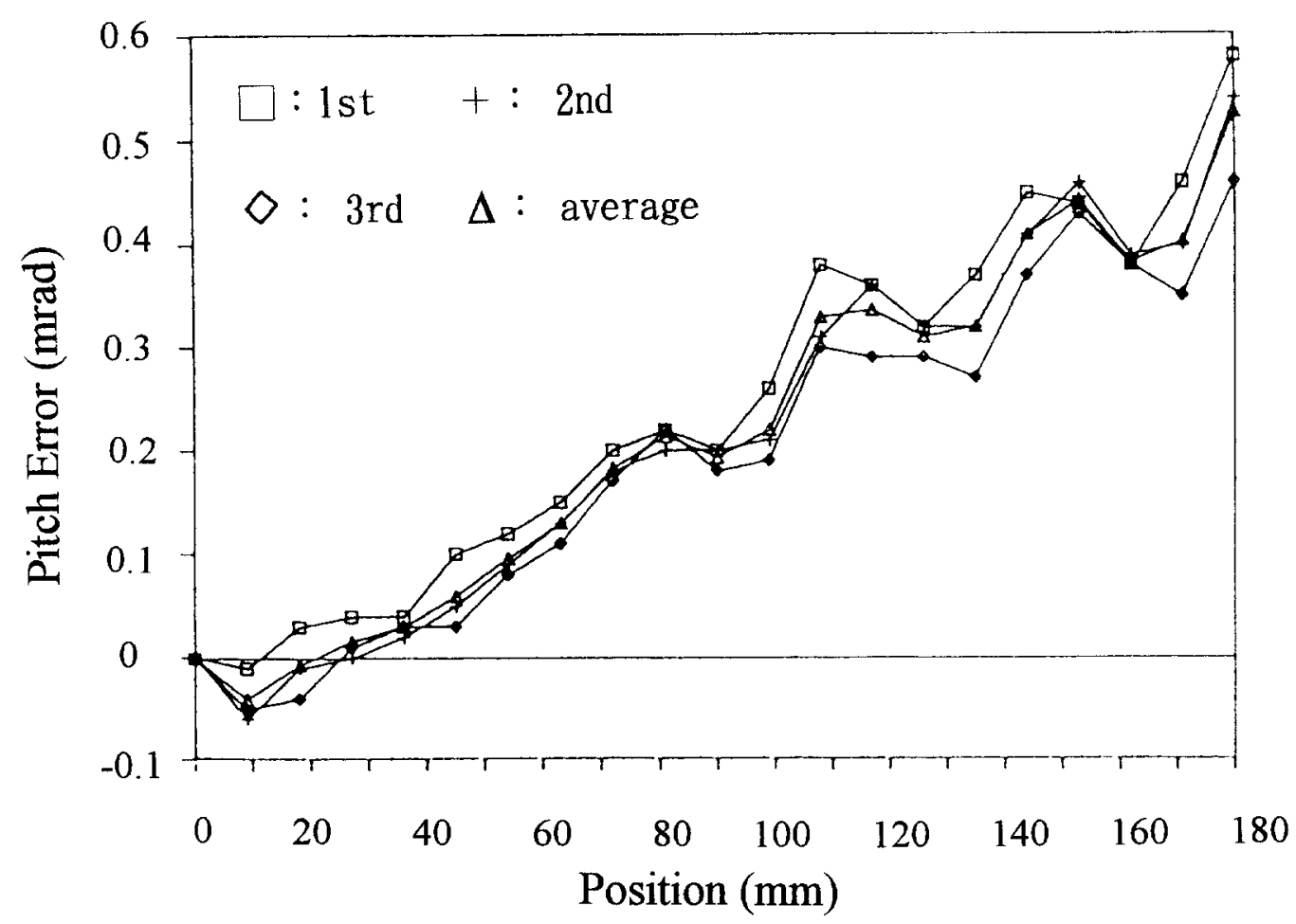

Figure 13 Pitch errors of the linear stage

grid lines and reflect onto the screen on the right hand side of Figure 10. The mean height of this distorted image line on the screen is chosen as the reference line for all the image lines of this plate. The linear stage then drives the plate in the X-direction to the next scanning position, which is the midpoint of the next two $X$ nodal points and the image data are taken. This process is repeated to the last scanning position. The plate is then rotated counterclockwise by $90^{\circ}$, and the second similar scanning process is executed.

From the first scanning process, the slope of each $j$ th target point of the $i$ th scanning line, in the $X$-direction, $\theta_{i, j}$ can be computed. The relative height of two consecutive points in the $X$-direction can be computed by the corresponding slope data, as shown in Figure 11, by the following equation

$$
d_{i, j}=L_{x} \tan \theta_{i-1, j}
$$

where $L_{x}$ is the step length of the grid in the $X$-direction. Then the height of each $j$ th target point along the $i$ th line relative to the first point of the same line can be accumulated from the first point as

$$
\begin{array}{rlrl}
Z_{i, j}=Z_{1, j}+\sum_{n=2}^{i} d_{n, j}, & & \\
\text { for } i=2 & \text { to } & A, \\
j & =1 & \text { to } & B
\end{array}
$$

From the second scanning process, the relative height of two consecutive points along the first $Y$-grid line can be obtained by the following equation.

$$
d_{1, j}=L_{y} \tan \theta_{1, j-1}, \text { for } \quad j=2 \text { to } B
$$

where $L_{y}$ is the step length of the grid in the $Y$-direction. Then the height of the $j$ th point of the first $Y$ line can be obtained as

$$
Z_{1, j}=Z_{1,1}+\sum_{m=2}^{j} d_{1, m}, \quad \text { for } j=2 \text { to } B
$$

Let the first node of the grid be the reference point of the surface. Its height $Z_{1,1}$ is assumed to be zero. The surface height of each node relative to this reference point can, thus, be obtained by substituting Equation (18) into Equation (16).

\section{The least-squares flatness errors}

The surface so far obtained is still not leveled. To compensate for this error, a mean plane of the surface must be found. Let the equation of the mean plane be $Z=A X+B Y+C$. Coefficients $A$, $B, C$ can easily be obtained by the conventional least-squares method. ${ }^{17}$ The flatness error of the inspected surface is then determined by the difference in heights between the highest peak and the lowest valley of all points with respect to the mean plane. 


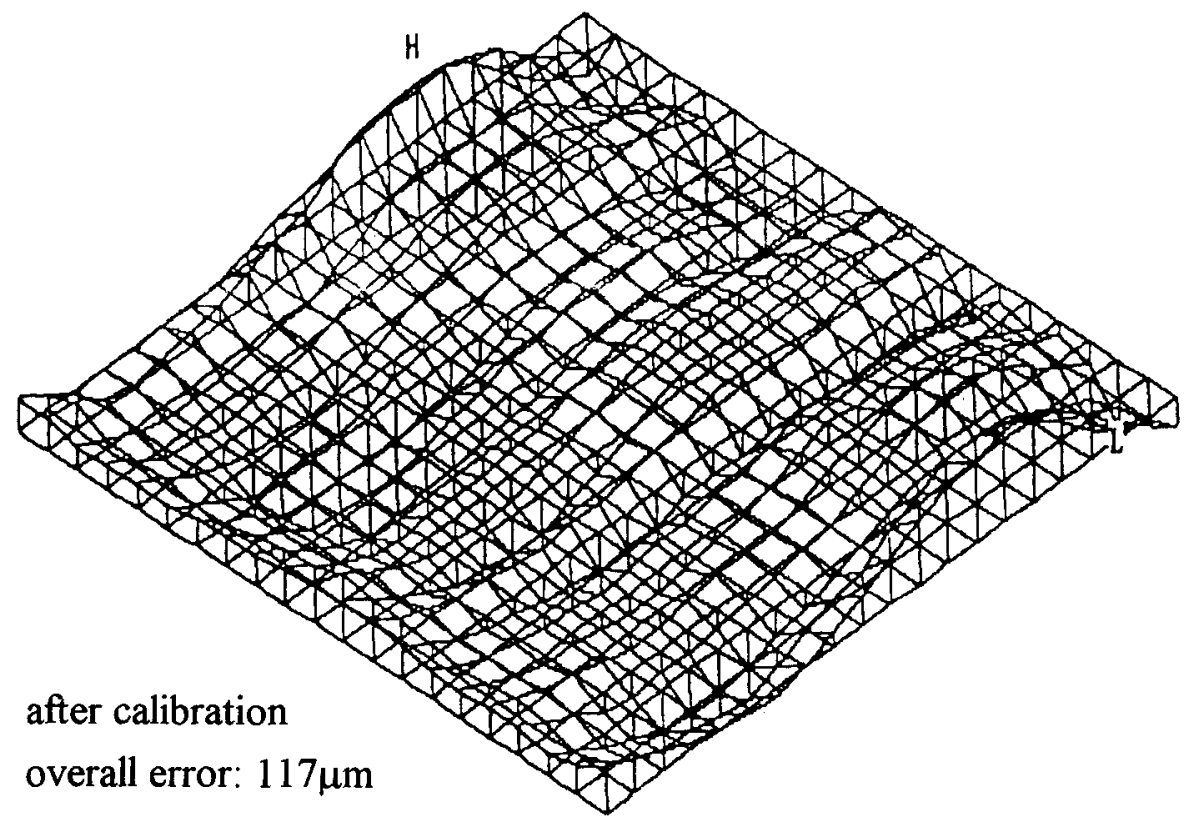

(A) Measured by PBSS

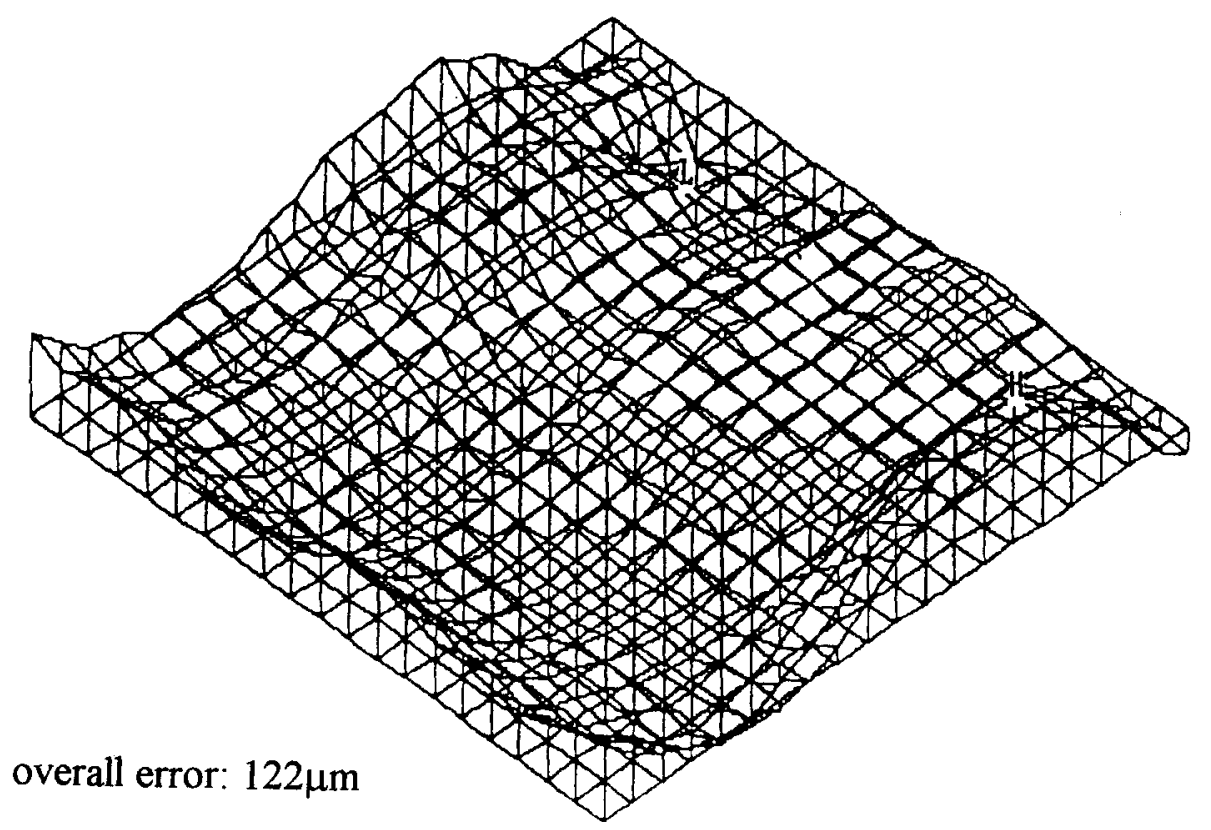

(B) Measured by CMM

Figure 14 Surface profile of the first specimen; (A) measured by PBSS; (B) measured by CMM

\section{System calibration}

There are three main sources of scale error for the system: the laser pointing stability, projection op- tics, and stage travel pitch. Each of these was investigated, and compensation was applied when necessary. Because the resolution of the system 


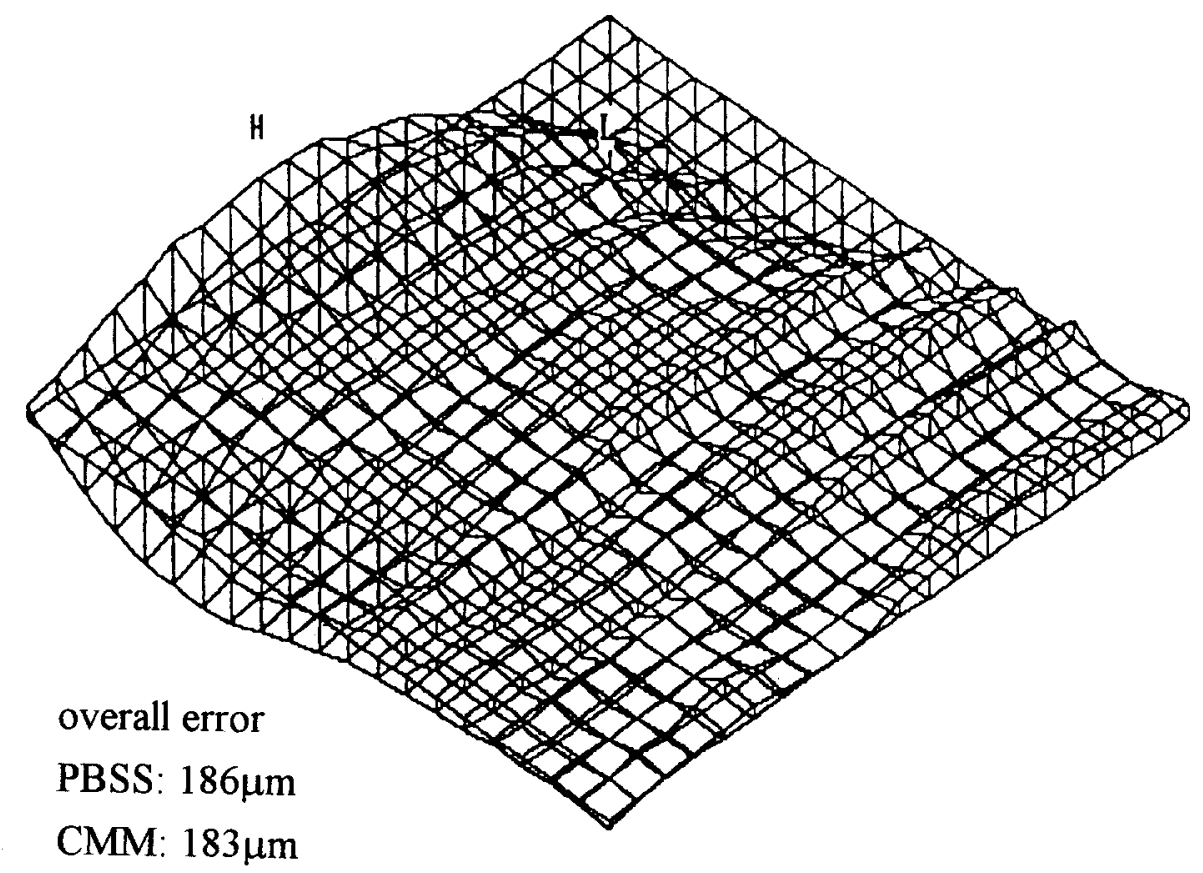

Figure 15 Surface profile of the second specimen

( 0.5 pixel) corresponds to an angular error of 0.3 mrad, errors much smaller than this were considered insignificant.

The angular drift, or pointing stability, of laser beams has received increasing attention, as applications require critical pointing stability. The light source adopted for use in this system is a $5 \mathrm{~mW} \mathrm{He}-\mathrm{Ne}$ laser model $\mathrm{U}-1325 \mathrm{P}$ made by Uniphase Co., with specified angular drift of 0.02 mrad after warm-up. A 14-hour test run was conducted in the laboratory. The spot movement on the screen was found within one pixel per hour. Because the entire duration of measurement by PBSS would not exceed 5 minutes per one plate of $20 \mathrm{~cm} \times 20 \mathrm{~cm}$ in size, the performance of pointing stability of this system was regarded acceptable.

The degree of parallelism of incident laser beams was also investigated by measuring the

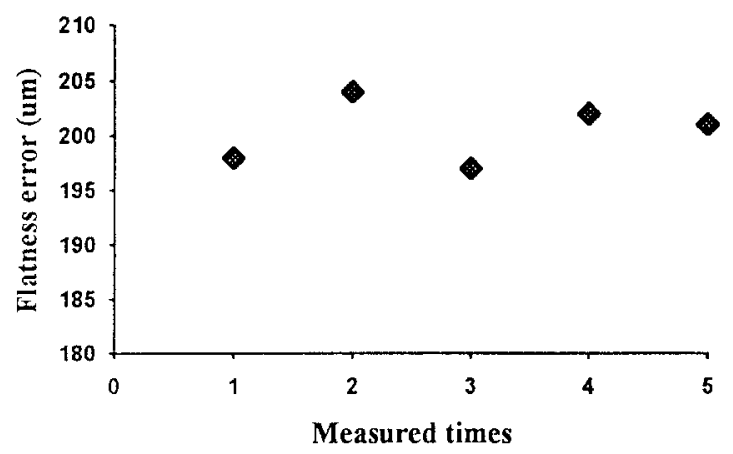

Figure 16 Repeatability test of PBSS length of the scanning line at different distances. The aplanatic meniscus lens was mounted on a five-axis gimbal optic mount. With a good adjustment of the lens, the parallelism of laser beams was quite satisfied.

The most critical error source, or the most sensitive factor to the systematic accuracy, found in this PBSS was the pitch error (angular movement of $X$-slope) of the linear stage. The pitch error at each stage position is directly superposed on the computed value of $X$-slope $\theta$ of the surface. Experimental calibration of this kind of error was done by an electronic level, as illustrated in Figure 12, Calibrated results showed, in Figure 13, the pitch error of the stage increased with the traveling distance. The maximum pitch error could reach up to $0.5 \mathrm{mrad}$. Therefore, at each stage position, this error has to be subtracted from Equation (14)

\section{Experimental results}

In the experiment, some SMC plates were used as specimens. The roughness value $(\mathrm{Ra})$ of the specimens varied from 0.2 to $0.4 \mu \mathrm{m}$. The surface profile of the first plate obtained by the PBSS method is shown in Figure 14a. Using the CMM to measure the same plate, its flatness profile is given in Figure $14 b$ with evaluated flatness error of 122 $\mu \mathrm{m}$. It is clearly seen both profiles are very similar in shape. Comparing these two profiles, a correction factor can be applied to convert the pixel value of Figure $14 a$ to micrometers. The correction 
factor determined in this experiment is 0.63 , which gives the minimum deviation between corresponding lines of the entire profile. With this correction factor, the flatness data as well as the flatness error of Figure $14 a$ could be realized quantitatively. Figure 15 presents the measured results of another plate by PBSS and CMM respectively. Again, not only the shapes, but also the flatness errors, are both quite close to each other. The time required to complete each test was only 5 minutes. Figure 16 shows the results of five runs PBSS test on the same plate in the duration of two hours. The repeatability was found to be within $5 \%$.

\section{Conclusions}

A parallel beam scanning system was developed in this work for the flatness measurement of medium-sized surface plates. The principal concept of the PBSS is to measure the slope of the target point first, rather than the direct surface height of that point. These slope data of the entire surface targets can then be converted to the corresponding height data of the target points. This concept coincides with the principle of normal flatness measurement by angles. A mathematical model was proposed for the analysis of measured data. Comparing with the results from the CMM, this PBSS method was found quite accurate and repeatable. Because the PBSS method is noncontact, automatic, fast, and low cost, it could be a useful tool for practical applications in industry, especially for online measurement of medium-sized surface plates.

Finally, it should be pointed out here that, the measurement system is only suitable for fairly flat and smooth surfaces that observe the law of reflection. If the incident light is predominately scattered rather than reflected, the scattered light may reduce the contrast between the reflected light and the background enough that the system may not work.

\section{References}

1 ANSI Y14.5M, Dimensioning and Tolerancing for Engineering Drawings, New York: American National Standards Institute, Inc. 1982

2 BS 308, Part 3, Geometrical Tolerance, British Standards Institute, London, 1972

3 ISO/R1101, 1983, Technical drawings-Geometrical tolerancing

4 Abdin, S. Z., Shenoy, R. K. and Visveswaran, M. E. "Investigation on granite as a material for metrology aids," Ann CIRP, 1978, 27 377-381

5 Burdekin, M. and Pahk, H. J. "The application of a microcomputer to the on-line calibration of the flatness of engineering surfaces," Proc Inst Mech Eng, 1989, 203, 127-137

6 Moore, W. R. Foundations of Mechanical Accuracy, The Moore Special Tool Company, Connecticut, 1970

7 "Calibration of a Surface Plate," Hewlett Packard Application Note 156-2

8 Galyer, J. F. W. and Shotbolt, C. R. Metrology for Engineers, 5th ed. London: Cassell, 1990

9 Kralovec, W. "Optical evaluation of long-term surface waviness," Proceedings of the 23rd Annual Conference, Reinforced Plastics/Composites Institute, The Society of the Plastics Industry, Inc., Section 1-C, 1968, 1-6

10 Hust, A. T. "Measurement aspects and improvement of surface profile in thin gauge mold SMC," Proceedings of the 37th annual conference, Reinforced Plastics/Composites Institute, the Society of the Plastics Industry, Inc., 1982, 11-15

11 Iseler, K. A. and Wilkinson, R. E. "A surface evaluation system for class A applications," Proceedings of the 39th annual conference, Reinforced Plastics/Composites Institute, the Society of the Plastics Industry, Inc., 1984, 16-19

12 Hupp, S. S. "A quantitative method for analysis of surfaces of molded SMC parts," Proceedings of the 43rd annual conference, Composites Institute, the Society of the Plastics Industry, Inc., 1988, 1-5

13 Hupp, S. S. and Hackett, T. B. "Measurement and characterization of adhesive bondline read-through," Proceedings of the 44th annual conference, Composites Institute, the Society of the Plastics Industry, Inc., 1989, 6-9

14 Hupp, S. S. and Hackett, T. B. "Quantitative analysis of surface quality for exterior body panels," SAE Paper SP743, 1988, 39-44.

15 Lin, H. Y. "Surface smoothness measurement on thin plates." Master's thesis, National Taiwan Univ., 1989

16 Brodmann, R. "Roughness form and waviness measurement by means of light-scattering," Prec Eng, 1986, 8, 221226

17 Scarr, A. J. "Use of the least-squares line and plane in the measurement of straightness and flatness," Proc Inst Mech Eng, 1968, 182, 531-536 